COMMENT. Executive functions may be defined as mental control cognitive processes that facilitate goal directed behavior. They include response inhibition, planning, cognitive flexibility/set shifting, working memory, and verbal fluency. Attention is defined as a set of processes that enhance cognitive, motor, and sensory processing. Sustained attention is the ability to maintain performance without distraction during continuous activities. Divided attention is the ability to simultaneously respond to multiple stimuli. The present study investigated all five executive functioning domains and attention in ADHD subtypes. The ADHD subtypes (inattentive and combined) are homogeneous in neuropsychological profiles except for the role of interference inhibition on working memory. Inhibition of interference predicts performance on working memory only in the inattentive subtype. The findings support the involvement of partially independent neuronal circuits that control inhibition and divided attention in ADHD. Right prefrontal cortex controls response inhibition, while left dorsolateral prefrontal cortex modulates divided attention.

Role of neuropsychological tests in diagnosis of ADHD. In a study performed in Columbia and at the University of Georgia, neuropsychological measures, including the continuous performance tests (CPT), had strong sensitivity but weak specificity when used in diagnosis of ADHD. (Pineda DA et al. Pediatr Neurol 2007;36:373-381). CPT is not recommended as a measure for clinical diagnosis of ADHD. CPT is sensitive to attention problems caused by medication, depression, ADHD and other causes, and is not specific for ADHD. Neuropsychological measures are important in planning interventions for the ADHD child with concomitant learning problems or academic underachievement.

\title{
METHYLPHENIDATE IN TREATMENT OF ADHD AND COMORBID CHRONIC TIC DISORDER
}

The safety and efficacy of immediate-release methylphenidate (MPH-IR) for the treatment of attention deficit hyperactivity disorder (ADHD) in children (ages 6-12 years) with Tourette's syndrome $(96 \%)$ or chronic motor tic disorder $(4 \%)$ were evaluated at State University of New York, Stony Brook. Seventy one children, in 2 cohorts, were treated with three doses of MPH $(0.1,0.3$, and $0.5 \mathrm{mg} / \mathrm{kg})$ and placebo twice daily for 2 weeks each, double-blind, and effects were assessed with a battery of parent, teacher, child, and physician-completed rating scales and laboratory tasks. MPH-IR effectively suppressed ADHD, oppositional defiant disorder, and peer aggression behaviors, without altering the overall severity of tic disorder or obsessive compulsive disorder. Dose-response was variable. Teacher ratings indicated that MPH-IR treatment decreased the frequency and severity of the tic disorder. The physician's 2-minute Tic/Habit Count showed an increase in simple but not complex motor movements $(0.3$ and $0.5 \mathrm{mg} / \mathrm{kg}>$ placebo). For the Simulated Classroom, the tic frequency was slightly higher for the $0.5 \mathrm{mg} / \mathrm{kg}$ dose. Adverse effects related to dose included heart rate and blood pressure increases and weight loss. MPH-IR is not contraindicated as a short-term therapy for children with ADHD and chronic tic disorder, but treatment should be carefully monitored to exclude possible tic exacerbation in susceptible individuals. (Gadow KD, Sverd J, Nolan EE, Sprafkin J, Schneider J. Immediaterelease methylphenidate for ADHD in children with comorbid chronic multiple tic disorder. J Am Acad Child Adolesc Psychiatry July 2007;46:840-848). (Respond: Dr Kenneth 
Gadow, Department of Psychiatry and Behavioral Science, Putnam Hall, South Campus, SUNY at Stony Brook, Stony Brook, NY 11794).

COMMENT. These authors recommend a carefully monitored trial of immediaterelease methylphenidate $(\mathrm{MPH})$ in the treatment of ADHD with comorbid chronic tic disorder (CTD). Larger doses of MPH resulted in minimal increased effectiveness, but with a greater likelihood of tic exacerbation and adverse effects on heart rate, blood pressure and weight. The improved tic control reported by teachers in the classroom, in contrast to the physicians' report of an increase in simple tics with larger doses, may be explained by a student's ability to suppress tics in the stigmatizing school environment. Evaluations in multiple environments are necessary to determine the true frequency and severity of a tic disorder. A non-stimulant such as atamoxetine may be an alternative initial drug of choice for the treatment of ADHD and comorbid CTD, but immediate-release MPH in conservative doses may be safe and effective.

\section{LANGUAGE DISORDERS}

\section{LANGUAGE DEVELOPMENT AND CSWSS EPILEPSY SYNDROME}

Linguistic and pragmatic skills (comprehension that is context dependent) were examined in 10 right-handed children and adolescents with the continuous spike-waves during slow sleep syndrome (CSWSS) and compared to that of a control population of 36 children, in a study at CHRU de Tours and Universite Francois Rabelais, Tours, France. Patients with CSWSS had lower scores in tests measuring lexical and pragmatic skills compared to controls, whereas oral comprehension was unaffected. Language impairment showed no improvement when patients were in remission and even after total disappearance of EEG anomalies. The language impairment profile of CSWSS is different from that of Landau-Kleffner syndrome, characterized by auditory and verbal agnosia with limited spoken language and loss of verbal comprehension. Evaluation of language skills and longterm outcome are important in the management of epileptic syndromes. (Debiais S, Tuller L, Barthez M-A et al. Epilepsy and language development; The continuous spike-waves during slow sleep syndrome. Epilepsia June 2007;48:1104-1110). (Reprints: Dr Caroline Hommet, Service de Neurologie, CHRU de Tours, 37044 Tours cedex, France).

COMMENT. Continuous spike-waves during slow sleep syndrome (CSWSS) is a childhood epilepsy characterized by EEG spike waves during at least $85 \%$ of slow sleep, and clinically, by neuropsychological and behavioral disorders. CSWSS and Landau-Kleffner syndrome are similar in age of onset, mild epilepsy, and severe neuropsychological disorders. The present study shows that both syndromes are associated with impairments in language development, but the acquired dysphasias have different profiles. It is postulated that the nature and severity of the neuropsychological and language deficits are determined by the site of paroxysmal activity during early cerebral and language development. 